\title{
Actividade física e bem-estar psicológico - perfil dos participantes no programa de exercício e saúde de rio maior
}

Luís Cid , Carlos Silva e José Alves

Escola Superior de Desporto de Rio Maior

Departamento de Psicologia e Ciências Sociais do Desporto

\author{
Cid, L.; Silva, C.; Alves, J.; Actividade física e
} bem-estar psicológico - perfil dos participantes no programa de exercício e saúde de rio maior Motricidade 3(2): 47-55

\section{Resumo}

Hoje em dia existem amplas evidências científicas de que a actividade física regular tem beneficios inquestionáveis para a saúde física e psicológica, que por sua vez são causadores de um impacto significativo no bem-estar geral do sujeito em todas as idades. Desta forma, foi objectivo principal do nosso trabalho, estabelecer um perfil psicológico dos sujeitos aderentes aos programas de exercício e saúde, baseado nas seguintes variáveis: motivação, estados de humor, depressão, ansiedade e stress. Para tal propósito, estudámos uma amostra composta por 70 indivíduos de ambos os sexos (67 femininos e 3 masculinos), com uma média de idades de $36.90 \pm 15.4$ anos (entre os 17 e os 71). Todos pertencem aos cinco centros de prática desportiva, inseridos no programa de exercício para a saúde "Mais Desporto, Mais Saúde", da Câmara Municipal de Rio Maior. Os instrumentos de avaliação utilizados para o efeito foram os seguintes: EMI-2 (Exercise Motivations Inventory); POMS (Profile of Mood States); DASS (Depression, Anxiety and Stress Scales). Com os resultados obtidos pudemos concluir que as principais razões que levam os indivíduos a aderir a este tipo de programas são os factores associados à saúde, ou seja, apresentam um perfil de uma pessoa que procura a actividade fisica para se manter saudável (e.g. Manter-

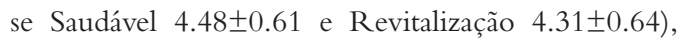
uma vez que esta lhe provoca sensação de vigor (i.e. bem-estar fisico ou mental). Este sentimento reflectese nos valores altos dos estados positivos de humor (e.g. vigor $2.28 \pm 0.87$ ), bem como, nos baixos valores encontrados nos níveis de depressão $(0.47 \pm 0.61)$, ansiedade $(0.49 \pm 0.58)$ e stress $(0.81 \pm 0.61)$.

Palavras-chave: Psicologia do exercício, bem-estar psicológico, exercício e saúde Data de submissão: Dezembro 2007

Data de Aceitação: Abril 2007

\section{Abstract}

Physical activity and psychological well-being - rio maior participants profile in the exercise and health program

Nowadays several scientific evidences tell us that the regular physical activity has unquestionable benefits for physical and psychological health, and that causes a significant impact in general well-being into people of all ages. The purpose of this study was to evaluate and establish a psychological profile of the adherence to exercise and health programs, based in the following variables: motivation, mood states, depression, anxiety and stress. For such, we studied a sample of 70 individuals, of both genders (67 females and 3 males), with $36.90 \pm 15.4$ years old of mean age (between 17 and the 71). All of them are participants of the five sport centers that compose the exercise and health programme "More Sport, More Health", of Rio Maior town council. The measurement instruments were the following: EMI-2 (Exercise Motivations Inventory); POMS (Profile of Mood States); DASS (Depression, Anxiety and Stress Scales). Results show that the main reason to engage individuals in this kind of programs are health related factors. In other words, individuals present a profile that seeks physical activity to be healthy (e.g. Being Healthy $4.48 \pm 0.61$ and Revitalization $4.31 \pm 0.64)$, because that gives sensations of vigor-activity (i.e. physical or mental strength). This feeling reflects the higher value of positive mood states (e.g. vigor 2.28 \pm 0.87 ), also the lower values of depression $(0.47 \pm 0.61)$, anxiety $(0.49 \pm 0.58)$ and stress $(0.81 \pm 0.62)$.

Keywords: Exercise psychology, psychological wellbeing, exercise and health 


\section{Introdução}

"A qualidade de vida é um conceito amplo que integra a noção de bem-estar ou felicidade (...) e o exercício físico habitual influencia essa qualidade de vida de diferentes formas" 4 , pp. 50

A grande diversidade dos problemas de saúde, com que habitualmente se debatem os países mais desenvolvidos, estão associados a alterações sistemáticas dos hábitos de vida, nos quais se inclui uma redução dos níveis de actividade física e exercício que conduzem a um estilo de vida cada vez mais sedentário. Hoje em dia, existe uma ampla evidência de que o exercício regular e moderado tem benefícios inquestionáveis para a saúde fisica, psicológica e social, podendo contribuir de forma significativa para o bem-estar geral do sujeito em todas as idades1,4,6,7,8,17,30.

Como se sabe, o interesse humano em relacionar a mente e o corpo não é recente, a mítica expressão "mens sana in corpore sano" é um fiel reflexo da saúde mental proporcionada pela actividade física e o desporto10. A procura do bem-estar psicológico e/ou equilíbrio emocional, muitas das vezes perdido devido às enormes exigências e pressões colocadas pela sociedade moderna, faz com que cada vez mais as pessoas adiram à prática de exercício físico como forma de escape da rotina quotidiana9. Por isso, quando alguém justifica o facto de ter aderido a um programa de actividade física, ouvimos constantemente dizer: "porque me faz sentir bem"; "porque me ajuda a combater o stress"; " porque me ajuda a libertar a tensão".

As evidências empíricas, vivenciadas pelos sujeitos que praticam exercício, são amplamente comprovadas pelo conhecimento científico que tem sido produzido nos últimos anos, tornando claro 芯 que a actividade física influencia positivamente não só a saúde física, como a psicológica, sendo a sua prática um factor fundamental em todas as fases do ciclo da vida humana, desde a infância até à idade mais avançada11. O crescente interesse pela investigação nesta área, levou a ISSP - International Society of Sport Psychology13, a publicar um documento relacionando a actividade física e os benefícios psicológicos, na qual vem expresso que o exercício a longo prazo está geralmente associado a uma redução dos níveis de ansiedade e stress, à diminuição de depressões, ao aumento da auto-estima e como factor gerador de efeitos emocionais positivos.

De facto, segundo diversos autores (e.g.4,6,8,32), as variáveis mais estudadas no âmbito da psicologia da actividade física e do exercício são: motivação, ansiedade, depressão, estados de humor, stress, auto-estima, personalidade, funcionamento cognitivo, atitudes e emoções.

Se por um lado, é fundamental conhecer as razões pelas quais as pessoas seleccionam determinadas actividades, nelas persistem e se lhes entregam com uma data intensidade5, na tentativa de compreender a aderência e/ou participação ao exercício - "tópicos que os investigadores mais tem dedicado o seu tempo e energia"6, pp.27, por outro, é igualmente indispensável entender quais são os factores psicológicos que podem estar associados à sensação de bem-estar e o modo como estes se relacionam com o exercício (i.e. antes, durante e após a sua prática) 1.

Como se sabe, de uma maneira ou de outra, todos nós tentamos procurar as razões (e.g. intrínsecas ou extrínsecas) pelas quais nos envolvemos, ou não, numa determinada actividade bem como os factores que influenciam a nossa continuidade nessa mesma actividade. Apesar do conhecimento generalizado sobre os efeitos positivos na saúde que a prática regular de exercício pode potenciar, uma esmagadora percentagem da população nas sociedades industrializadas é sedentária (i.e. cerca de $70 \%$ ) ou abandona a prática nos primeiros seis meses (i.e. cerca de 50\%), dando a entender que esses benefícios não são razões suficientes para 
Actividade física e bem-estar psicológico - perfil dos participantes no programa de exercício e saúde de rio maior Luís Cid , Carlos Silva e José Alves

que realizem actividade fisica 1,19.

Para além de preocupantes, os factos apresentados tornam clara a necessidade de se continuar a investigar nesta área, justificando a integração de novos modelos e implementação de novas estratégias na abordagem aos programas de exercício. Neste domínio a Psicologia aplicada pode ter um papel preponderante de acção.

$A$ actividade física não pode continuar a ver vista como uma simples forma de gastar calorias, por razões puramente estéticas, mas sim, como um meio para a promoção da saúde a todos os níveis. Este princípio, aceite por toda a comunidade científica, integra a noção de que o bem-estar psicológico está associado aos índices de participação em programas de exercício por parte dos sujeitos6. De acordo com Vasconcelos-Raposo30, este conceito é visto como uma expressão da felicidade, reflectindo um processo interno que tem como meta principal a atribuição de significado ou valor a aspectos relacionados com a qualidade de vida. Segundo Dosil10, esta representa um conjunto de padrões de conduta que caracterizam a maneira de viver de um sujeito e que estão directamente vinculados à satisfação das suas necessidades.

Desta forma, se partirmos do princípio que as pessoas se motivam a fazer aquilo que lhes permite satisfazer as suas necessidades, o estudo do perfil psicológico dos praticantes torna-se numa pedra basilar na organização e orientação do processo do seu envolvimento com a prática da actividade física. O conhecimento produzido numa avaliação neste domínio pode ajudar à promoção de um clima motivacional positivo, sensação de felicidade e satisfação pessoal, ou seja, a qualidade de vida da qual faz parte o bem-estar psicológico.

Assim sendo, os objectivos a que nos propomos são os seguintes: 1) Estabelecer um perfil psicológico dos aderentes a um programa de exer- cício para a saúde "Mais Desporto, Mais Saúde" de Rio Maior; 2) Fornecer algumas indicações no sentido da adequação dos programas de exercício às necessidades dos seus praticantes;

\section{Metodologia}

\section{Amostra}

A nossa amostra é constituída por 70 sujeitos de ambos os sexos (i.e. 67 femininos e 3 masculinos), com uma média de idades de 36,9 \pm 15,4 anos (i.e. variando entre os 17 e os 71). Todos pertencem aos 5 centros de prática desportiva, inseridos no programa de exercício para a saúde "Mais Desporto, Mais Saúde", da Câmara Municipal de Rio Maior (ver Franco e Moutão12), cujas actividades principais são no âmbito do fitness (i.e. aeróbica e step). Como informação complementar, podemos dizer que os sujeitos têm uma média de 2,2 \pm 1,4 anos de prática de actividade física regular, à qual dedicam uma média de 1,7 $\pm 0,8$ horas por semana e estão no programa pelo segundo ano consecutivo.

\section{Instrumentos}

Para avaliar os motivos para a prática desportiva ou as razões pessoais para a participação em programas de exercício físico, foi utilizado o Exercise Motivations Inventory (EMI-2), traduzido e validado por Alves e Lourenço2. O Questionário de Motivação para o Exercício (QME), designação pela qual ficou conhecido, é constituído por 51 afirmações, que representam 14 dimensões que se podem agrupar em cinco grupos de motivos (Tabela 1). As respostas são dadas numa escala tipo Lickert de 6 pontos $(0=$ "nada verdadeiro para mim" até ao $5=$ "completamente verdadeiro para mim”). 


\begin{tabular}{ll} 
Tabela 1 - Dimensões do EMI-2 & \\
\hline Grupos de Motivos & Dimensões (EMI-2) \\
\hline Psicológicos & Stress; Revitalização; Prazer; Desafio \\
Inter-pessoais & Reconhecimento Social; Afiliação; Desafio \\
Saúde & Saúde; Doença; Manter-se Saudável \\
Corporais & Peso; Aparência \\
Condição Física & Agilidade; Força/Resistência \\
\hline
\end{tabular}

Para avaliar os estados de humor dos indivíduos foi utilizado o Perfil de Estados de Humor (POMS), traduzido e validado porViana,Almeida e Santos31, do original Profile of Mood States (POMS). Este instrumento é composto por 42 itens aos quais os sujeitos têm de responder numa escala tipo Lickert de 5 pontos $(0=$ "nada" até ao 4 = "muitíssimo"), sobre a forma como se sentiram nos últimos dias. Posteriormente estes itens são agrupados em 6 dimensões (tabela 2).

\section{Procedimentos}

A recolha dos dados foi efectuada durante o primeiro mês após o início das actividades lectivas do programa e depois da obtenção do consentimento informado por parte dos sujeitos para participarem neste estudo.

Todos os instrumentos de avaliação utilizados foram aplicados em condições semelhantes (i.e. em grupos pequenos) e de acordo com os protocolos previamente estabelecidos (i.e. as recol-

\begin{tabular}{ll}
\hline Tabela 2 - Dimensões do POMS & \\
\hline Dimensão & Definição \\
\hline Tensão & Estado de tensão músculo-esquelética e preocupação. \\
Depressão & Estado emocional de desânimo, tristeza, infelicidade e solidão. \\
Hostilidade & Estado de humor de cólera e antipatia relativamente aos outros. \\
Vigor & Estado de energia e vigor físico e psicológico. \\
Fadiga & Estado de cansaço, inércia e baixa energia. \\
Confusão & Estado de confusão e baixa nitidez. \\
\hline
\end{tabular}

Para avaliar os níveis emocionais dos sujeitos foi utilizada a versão portuguesa (reduzida) do DASS - Depression, Anxiety and Stress Scale, desenvolvido por Lovibond \& Lovibond15. Este questionário é composto por 21 itens, aos quais se responde através de uma escala tipo Lickert de 4 pontos $(0=$ "nada se aplicou a mim" até ao 3 = "aplicou-se a mim muito, ou a maior parte do 空 tempo"). Posteriormente os itens são agrupados 菏 em 3 dimensões (tabela 3). has foram previamente agendadas com os participantes). Para tal, foi disponibilizado em cada centro de prática um espaço próprio adequado para o efeito, de modo a que os indivíduos não se sentissem estranhos com a situação e, ao mesmo tempo, pudessem estar concentrados durante o preenchimento dos questionários.

\section{Estatística}

Após a recolha e inserção dos dados no aplicativo SPSS (Statistical Package for the Social Sciences), versão 14.0, procedeu-se à análise descri- 
Actividade física e bem-estar psicológico - perfil dos participantes no programa de exercício e saúde de rio maior Luís Cid , Carlos Silva e José Alves

\begin{tabular}{lc}
\hline Tabela 3 - Dimensões do DASS \\
\hline Dimensão & Definição \\
Depressão & Associado a sintomas negativos do humor (e.g. tristeza) \\
Ansiedade & Associado a sintomas de pânico e medo (e.g. tremuras) \\
Stress & Associado a sintomas de tensão e irritabilidade (e.g. irritação) \\
\hline
\end{tabular}

tiva dos mesmos. Para tal, apenas foram utilizadas medidas de tendência central e de dispersão (i.e. médias e desvios-padrão).

\section{Resultados}

\section{Motivação}

$\mathrm{Na}$ tabela 4, podemos observar quais os motivos (mais e menos importantes), para a prática de actividade física, apresentados pelos sujeitos da nossa amostra. valorizados. Por outro lado, o "desafio" (i.e. procura de novos objectivos e orientações pessoais), "aparência" (i.e. aspecto corporal), "competição" (i.e. gostar de competir), "saúde" (i.e. ajudar a combater uma doença) e "reconhecimento social" (i.e. obter reconhecimento e prestigio social), foram os motivos menos valorizados.

\section{Estados de Humor}

$\mathrm{Na}$ tabela 5, são apresentados os resultados relativos aos estados de humor dos sujeitos da nossa amostra.

\begin{tabular}{lll}
\hline \multicolumn{2}{l}{ Tabela 4 - Motivação dos Aderentes ao Programa de Exercício e Saúde } \\
\hline Ordem & Dimensões & Média \pm Desvio Padrão \\
1 & Manter-se Saudável & $4,48 \pm 0,61$ \\
2 & Revitalização & $4,31 \pm 0,64$ \\
3 & Agilidade & $4,11 \pm 0,74$ \\
4 & Prazer & $3,98 \pm 0,79$ \\
5 & Força e Resistência & $3,81 \pm 0,84$ \\
6 & Doença & $3,80 \pm 1,07$ \\
7 & Stress & $3,46 \pm 0,79$ \\
8 & Peso & $3,38 \pm 1,03$ \\
9 & Afiliação & $3,04 \pm 0,88$ \\
10 & Desafio & $2,81 \pm 0,99$ \\
11 & Aparência & $2,45 \pm 1,05$ \\
12 & Competição & $2,18 \pm 1,31$ \\
13 & Saúde & $1,90 \pm 1,48$ \\
14 & Reconhecimento Social & $1,30 \pm 1,12$ \\
\hline
\end{tabular}

Como podemos constatar, na avaliação efectuada, os motivos "manter-se saudável" (i.e. manter uma boa saúde), "revitalização" (i.e. sensação de bem-estar fisico e mental) e "agilidade" (i.e. condição fisica, boa flexibilidade), foram os mais
Como se pode observar, os resultados indicam que a dimensão mais valorizada pelos sujeitos foi o "vigor" (i.e. estado de humor positivo representativo de energia e vigor físico e/ou psicológico), embora o valor médio apresentado (i.e. $2.28 \pm 0.87)$ corresponda ao nível de moderado. 


\begin{tabular}{lll}
\hline \multicolumn{3}{l}{ Tabela 5 - Estados de Humor dos Aderentes ao Programa de Exercício e Saúde } \\
\hline Ordem & Dimensões & Média \pm Desvio Padrão \\
\hline 1 & Vigor & $2,28 \pm 0,87$ \\
2 & Tensão & $1,41 \pm 0,45$ \\
3 & Fadiga & $1,23 \pm 0,76$ \\
4 & Confusão & $1,07 \pm 0,40$ \\
5 & Hostilidade & $0,89 \pm 0,64$ \\
6 & Depressão & $0,47 \pm 0,45$ \\
\hline
\end{tabular}

Por outro lado, as dimensões "hostilidade" (i.e. estado de humor negativo significativo de mau estar na relação com os outros) e "depressão" (i.e. estado emocional de desânimo, tristeza, infelicidade e solidão) são as menos valorizadas.

\section{Stress, Ansiedade e Depressão}

De acordo com a tabela 6 , podemos constatar que os sujeitos apresentaram níveis baixos de ansiedade, stress e depressão o que de certa forma vem confirmar os resultados obtidos anteriormente na avaliação dos estados de humor.
saudável.Estes resultados parecem ser consistentes com alguns estudos realizados recentemente na área da actividade fisica, com amostras semelhantes à nossa (e.g.3,14,16,18,20,29). De facto, os motivos mais importantes de adesão e participação a programas de exercício, estão fortemente relacionados com as questões associadas à saúde e bem-estar dos sujeitos. De acordo com Moutão, Alves e Silva18 e Alves, Romero e Moutão3, estas razões ganham uma importância acrescida à medida que a idade avança. Desta forma, tal como sugerem estes autores, é necessário avaliar

\begin{tabular}{lll}
\hline \multicolumn{2}{l}{ Tabela $6-$ Sintomas (negativos) dos Aderentes ao Programa de Exercício e Saúde } \\
\hline Ordem & Dimensões & Média \pm Desvio Padrão \\
\hline 1 & Stress & $0,81 \pm 0,62$ \\
2 & Ansiedade & $0,49 \pm 0,58$ \\
3 & Depressão & $1,23 \pm 0,76$ \\
\hline
\end{tabular}

Como se verifica, os resultados nestas variáveis são pouco expressivos em termos de valores absolutos. Relembramos que a pontuação em cada dimensão pode variar entre 0 e 3 .

\section{Discussão}

Tendo em linha de conta os objectivos traçados, podemos concluir, que as principais razões : que levam os indivíduos a aderir e manter-se no programa, tem por base os factores associados à saúde, ou seja, apresentam um perfil de uma pesiิ soa que procura a actividade fisica para se manter as repercussões proporcionadas pelos programas de actividade fisica a todos os níveis (i.e. psicológicas, sócias, emocionais, saúde e bem-estar) e não apenas pelos ganhos ao nível da condição fisica e estética corporal.

Por outro lado, e ao que tudo indica, o exercício fisco pode desencadear a sensação de vigor que conduz ao bem-estar psicológico. Este sentimento, parece que se reflecte igualmente no aumento dos estados positivos de humor e numa diminuição dos níveis de depressão, ansiedade e stress. Os resultados obtidos na nossa avaliação são concordantes com o "perfil iceberg" de Morgan que descreve as alter- 
Actividade física e bem-estar psicológico - perfil dos participantes no programa de exercício e saúde de rio maior Luís Cid , Carlos Silva e José Alves

ações dos estados de humor induzidas pela actividade fisica. Este perfil é caracterizado por diversos autores (e.g.4,6), por um aumento dos estados de humor positivos (e.g. vigor) e uma diminuição dos negativos (e.g. tensão, depressão, hostilidade, fadiga e confusão). Alguns estudos realizados recentemente em Portugal, com amostras semelhantes àquela que foi utilizada por nós, demonstram esta tendência de forma clara (e.g.21,22,26). Veja-se por exemplo, o trabalho de Romero e Alves26, que ao implementarem um programa de actividade fisica para idosos (i.e. programa com a duração de três meses), para analisarem a influência do exercício fisico nos estados de humor, concluíram que o "perfil iceberg" não só se mantém no final do programa, como se acentuam as diferenças entre os estados positivos e negativos de humor.

De acordo com as meta-análises realizadas por Biddle e Mutrie6, a relação inversa entre o exercício, a depressão e a ansiedade, que na maioria das vezes é induzida pela acumulação do estado de stress, parece ser suportada por inúmeros estudos. De facto, alguns trabalhos realizados entre nós parecem suportar de forma consistente a avaliação operada na presente investigação (e.g.23,24,25,27).

Neste sentido, será fundamental que as actividades se desenrolem com uma orientação especial e uma preocupação constante com a saúde das pessoas, estimulando o desenvolvimento, cada vez mais sustentado, dos hábitos de actividade fisica diária e de uma prática desportiva que proporcione a satisfação das suas necessidades individuais. A grande questão que se coloca agora é: como se poderá operacionalizar um programa de actividade fisica de modo a potenciar ao máximo os beneficios do foro mental, induzidos pelo exercício? A resposta a esta questão é-nos fornecida por diversas fontes.

Segundo a ISSP13, para se beneficiar dos efeitos psicológicos da actividade fisica há que alternar o exercício do tipo anaeróbico com o aeróbico. No entanto, deve-se dar prioridade à actividade aeróbica (e.g. jogging, footing, natação, ciclismo) de uma forma não competitiva e menos intensa, privilegiando, sempre que possível, as actividades em grupo e de contacto com a natureza. Sessões de 20 a 60 minutos, de 3 a 5 vezes por semana, com uma intensidade entre os 60\%-90\% da FCMax são os factores chave para que o exercício fisico possa originar benefícios psicológicos mais consistentes4,8,9,10,28,32.

De acordo com algumas fontes, citadas por Dosil10, durante a elaboração de um programa de exercício, é fundamental ter em linha de conta uma série de indicações para que se possa garantir o seu êxito. As mesmas devem ser dirigidas à conduta dos sujeitos face à actividade física: no incentivo (e.g. educação para o exercício através da criação de um clima motivacional positivo e actividades atractivas - avaliação das expectativas e custos-beneficios), na facilitação (e.g. criação de infra-estruturas pessoais e de uma rede de suporte social para o exercício através da organização e gestão do tempo e das actividades), no controlo (e.g. afirmação de um contrato comportamental através de uma programação das actividades e de estabelecimento de metas) e no reforço (e.g. regulação e/ou auto-regulação da actividade através do ajustamento dos programas, reformulação dos objectivos e técnicas cognitivas, que permitam aumentar e/ou reforçar a sensação de bem-estar geral).

Em suma, tal como nos demonstram as evidências cientificas, é fundamental ter presente que o exercício físico é imprescindível para a saúde do ser humano, contribuindo de forma decisiva para o seu bem-estar fisico, social e psicológico28. No entanto, para que as pessoas nele participem e se mantenham, é necessário encontrar novos caminhos com diferentes abordagens. Neste domínio a psicologia da actividade fisica e do exercício pode ter um papel decisivo a desempenhar. 


\section{Correspondência}

Luís Cid

Escola Superior de Desporto de Rio Maior

Departamento de Psicologia e Ciências Sociais do Desporto

Rua José Pedro Inês Canadas, Lote $1-\mathrm{R} / \mathrm{ch}$

2040-326 Rio Maior

luiscid@esdrm.pt

\section{Referências}

1.Alves J (2005). Exercício e saúde: adesão e efeitos psicológicos. Psychologica 39:57-73;

2. Alves J, Lourenço A (2003). Tradução e adaptação do Questionário de Motivação para o Exercício (Exercise Motivation Inventory2 - EMI2). Desporto.investigação \& Ciência 2: 3-11;

3. Alves J, Romero F, Moutão J (2005). Motivations of portuguese elderly to exercise. In: Morris T (Ed). Proceedings of the ISSP 11th World Congress of Sport Psychology. Sydney: ISSP (CD-Rom);

4. Berger B, Pargman D, Weinberg R (2002). Foudations of Exercise Psychology. Morgantown: Fitness Information Technology.

5. Biddle S (1995). Exercise motivation across the life span. In: Biddle $S$ (Ed). European Perspectives on Exercise and Sport Psychology. Champaign: Human Kinetics, 3-25;

6. Biddle S, Mutrie N (2001). Psychology of Physical Activity: Determinants, well-being and interventions. London: Routledge, Taylor \& Francis Group; 7. Bueno A (2002). Psicología del ejercicio y bienestar. In: Serpa S, Araújo D (Eds). Psicología do Desporto e do Exercício. Lisboa: Edições FMH, 87103;

8. Buckworth J, Dishman R (2002). Exercise Psychology. Champaign: Human Kinetics;

9. Cruz J, Machado P, Mota M (1996). Efeitos e ㅇ․․ beneficios psicológicos do exercício e da actividade 慇 fisica. In: Cruz J (Ed). Manual de Psicologia do Desporto. Braga: Sistemas Humanos e Organizacionais, if $91-116$;
10. Dosil J (2004). Psicologia de la Actividad Física y del Deporte. Madrid: McGraw Hill.

11. Forum Mondial (1995). Physical activity, health and well-being. In: Forum mondial sur l'activité physique et le sport. Québec: Forum mondial sur l'activité physique et le sport.

12. Franco S, Moutão J (2003). Programas de promoção de saúde "Mais Desporto, Mais Saúde". In: Seabra A, Catela D, Romero F, Moutão J, Pimenta N, Santos R, Franco S (Eds). Investigação em Exercício e Saúde. Rio Maior: Edições ESDRM, 181-187;

13. Internacional Society of Sport Psychology (1992). Physical activity and psychological benefits. A position statement. The Sport Psychologist 6:199203;

14. Lourenço A,Alves J, Silva A (2003). Motivações para a aderência ao exercício fisico em populações especiais. In: Seabra A, Catela D, Romero F, Moutão J, Pimenta N, Santos R, Franco S (Eds). Investigação em Exercício e Saúde. Rio Maior:Edições ESDRM, 157-166;

15. Lovibond P, Lovibond S (1995). Manual for the Depression Anxiety Stress Scales. Sydney: Psychology Foundation of Australia;

16. Macedo, J. e Silva, C. (2005). Motivação para a prática de actividade fisica no Concelho de Rio Maior. In:Vitorino A, Ramires A, Borrego A, Silva C, Martins J, Alves J, Cid L, Gouveia M, Almeida P, Sobreiro P (Eds). Actas do Congresso Internacional de Psicologia do Desporto e da Actividade Física. Rio Maior: Edições ESDRM (CD-Rom);

17. Mota J (2003).Actividade fisica e saúde na população infanto-juvenil. Referências e reflexões. In: Seabra A, Catela D, Romero F, Moutão J, Pimenta N, Santos R, Franco S (Eds). Investigação em Exercício e Saúde. Rio Maior: Edições ESDRM, 8-19;

18. Moutão J, Alves J, Silva C (2003). Motivos para a prática de exercício fisico. In: Seabra A, Catela D, Romero F, Moutão J, Pimenta N, Santos R, Franco S (Eds). Investigação em Exercício e Saúde. Rio Maior: Edições ESDRM, 201-210; 
Actividade física e bem-estar psicológico - perfil dos participantes no programa de exercício e saúde de rio maior Luís Cid , Carlos Silva e José Alves

19. Moutão J,Alves J (2004). Diferenças de motives para a prática de actividades de fitness tendo em conta o género. In: Dosil J, Prieto D (Eds). Actas do $1^{\circ}$ Congresso Galego-Português de Psicologia da Actividade Física e do Desporto. Pontevedra: UniversidadVigo, 22-27;

20. Moutão J,Alves J (2005). Motivos para a prática de actividades de fitness. In:Vitorino A, Ramires A, Borrego A, Silva C, Martins J, Alves J, Cid L, Gouveia M,Almeida P, Sobreiro P (Eds). Actas do Congresso Internacional de Psicologia do Desporto e da Actividade Física. Rio Maior: Edições ESDRM (CD-Rom);

21. Moutão J, Dutra D, Romano M, Barata N (2006). Estados de humor em idosos participantes e não participantes num programa de actividade fisica. In: Palmeira A (Ed). Livros de Resumos do Simpósio Exercício, Desporto e Saúde: Sinergias da Psicologia e Medicina. Lisboa: Universidade Lusófona (CD-Rom);

22. Moutão J, Cid L, Silva H, Alves J (in press). Effect of two different cardiovascular training protocols on mood states of exercisers. In:Actas do 12th European Congress of Sport Psychology: Sport and Exercise Psychology - Bridges between disciplines and cultures. Athos Palace, Halkidiki;

23. Nunes P, Palmeira A (2006). Psychological effects of exercise: A study of the association with psychosocial adherence variables in adults. In: Palmeira A, Araújo D, Passos P (Eds). Book of Abstracts 3rd Annual Metting ENYSSP Workshop 2006. Lisboa: Universidade Lusófona (CD-Rom);

24. Palmeira A, Martins S, Pombo R, Krug P. (2006). Can exercise by itself promote subjective well-being to everyone? Interactions between variables from the transtheoretical model and subjective well-being. In: Palmeira A, Araújo D, Passos P (Eds). Book of Abstracts 3rd Annual Metting ENYSSP Workshop 2006. Lisboa: Universidade Lusófona (CD-Rom); 25. Pinto C, Martins S, Palmeira A (2006). Study of physical activity effects on levels of stress, depression, anxiety and emotions in a college environment. In: Palmeira A, Araújo D, Passos P (Eds). Book of Abstracts 3rd Annual Metting ENYSSP Workshop 2006. Lisboa: Universidade Lusófona (CD-Rom); 26. Romero F, Alves J (2004). Efectos psicológicos de un programa combinado de ejercicio fisico y de educación nutricional en una población de diabéticos. In: Dosil J, Prieto D (Eds). Actas do $1^{\circ}$ Congresso Galego-Português de Psicologia da Actividade Física e do Desporto. Pontevedra: Universidad Vigo, 38-52;

27. Salvador M, Palmeira A (2006). Beneficios psicológicos do exercício para os idosos. In: Palmeira A (Ed). Livros de Resumos do Simpósio Exercício, Desporto e Saúde: Sinergias da Psicologia e Medicina. Lisboa: Universidade Lusófona (CD-Rom);

28. Samulski D (2002). Psicologia do Esporte. S.Paulo: Editora Manole;

29. Silva C, Martins S, Borrego C (2006). Motivação para a prática de actividade fisica em ginásios de Leiria. In: Almeida P (Eds). Actas do V Congresso Luso-Espanhol de Psicologia do Desporto e da Actividade Física. Lisboa: ISPA (CD-Rom);

30. Vasconcelos-Raposo J (2004). Bem-estar psicológico, prática de exercício físico, auto-estima e satisfação corporal. In: Dosil J, Prieto D (Eds). Actas do $1^{\circ}$ Congresso Galego-Português de Psicologia da Actividade Física e do Desporto. Pontevedra: UniversidadVigo, 1-15;

31. Viana M, Almeida P, Santos R (2001). Adaptação portuguesa da versão reduzida do perfil de Estados de Humor - POMS. Análise Psicológica 19(1):77-92;

32. Weinberg R, Gould D (1995). Foundations of Sport and Exercise Psychology. Champaign: Human Kinetics; 